Liverpool Preprint: LTH 388

hep-lat/9611011

8 November 1996

\title{
Hybrid mesons from quenched QCD
}

\author{
UKQCD Collaboration \\ P. Lacock, C. Michael \\ Theoretical Physics Division, Department of Mathematical Sciences, University of Liverpool, \\ Liverpool, L69 3BX, U.K. \\ P. Boyle, P. Rowland \\ Department of Physics \& Astronomy, University of Edinburgh, Edinburgh EH9 3JZ, Scotland
}

\begin{abstract}
We use lattice methods to evaluate from first principles the spectrum of hybrid mesons produced by gluonic excitations in quenched QCD with quark masses near the strange quark mass. For the spin-exotic mesons with $J^{P C}=1^{-+}, 0^{+-}$, and $2^{+-}$which are not present in the quark model, we determine the lightest state to be $1^{-+}$with mass of $2.0(2) \mathrm{GeV}$.
\end{abstract}

One of the goals of quantitative studies of QCD is to determine the masses and properties of states which are not allowed in the simple quark model because they contain gluonic excitations. The prototype for such a state is the glueball and accurate lattice studies [1, 2] have been able to pinpoint the relevant mass range for experimental study of the scalar glueball as around 1.6 $\mathrm{GeV}$. Another important area is the study of hybrid mesons which are $q \bar{q}$ mesons with gluonic excitation. Because the gluonic excitation can introduce angular momentum, the most clear cut signal for a hybrid meson is to search for $J^{P C}$ quantum numbers not allowed in the quark model. These include $J^{P C}=1^{-+}, 0^{+-}$, and $2^{+-}$. Again lattice QCD is capable, from first principles, of establishing the masses of these states.

In full QCD, the quark pair creation and anihilation processes will lead to the mixing of glueballs with $q \bar{q}$ mesons and it is thus appropriate to establish the glueball spectrum with these processes turned off - the quenched approximation. This then acts as a guide for mixing studies. For hybrid mesons, however, the quenched approximation already allows mixing between $q \bar{q}$ mesons and hybrid mesons if their $J^{P C}$ quantum numbers are non-exotic. This will be quite difficult to untangle in lattice studies, so we focus instead on the exotic $J^{P C}$ cases. Any mixing for these exotic $J^{P C}$ states would have to be with $q \bar{q} q \bar{q}$ mesons and such mixing is turned off in the quenched approximation. Thus, in the quenched approximation, we are able 
to determine unambiguously the exotic hybrid energy levels given by the full non-perturbative gluonic interaction.

The spectrum of hybrid mesons has been explored in lattice QCD for many years [3, 4] in the context of heavy quarks. In the quenched approximation, the lightest hybrid level is found to be degenerate and to contain spin-exotic mesons with $J^{P C}=1^{-+}, 0^{+-}$, and $2^{+-}$. Since the quenched approximation does not reproduce exactly the experimental $c \bar{c}$ and $b \bar{b}$ spectra, the prescription of increasing the short distance component in the quenched approximation potential yields the lattice prediction [四] for the lightest hybrid meson excitation to be at $4.19(15) \mathrm{GeV}$ for $c \bar{c}$ and $10.81(25) \mathrm{GeV}$ for $b \bar{b}$. These energy values lie above the open $D \bar{D}$ and $B \bar{B}$ thresholds. An alternative procedure, within the quenched approximation, is to focus [5] on the energy difference between the hybrid meson and the $B \bar{B}$ threshold. This suggests that the lowest hybrid level may lie below the threshold. If the hybrid states were to lie below these thresholds, then they would be narrow resonances and so much clearer to detect experimentally. These lattice results are for the quenched approximation and do not include mixing with 4-quark states. They also they do not include spin-orbit effects which may be significant for quarks of finite mass. This is one motivation for exploring hybrid levels on a lattice with realistic quark masses.

There have been many experimental claims of signals for $J^{P C}$ exotic mesons in light quark spectroscopy (by light we mean composed of $\mathrm{u}$, d, s quarks). None of these claims are overwhelming [6, [] and guidance from QCD is sorely needed, as it was for glueball exploration. With present computational resources, it is possible to explore this and here we present the results of the first systematic study. Details of our lattice techniques and our preliminary results from much lower statistics have been published earlier [8]. Another lattice collaboration is also engaged in a study of this area [9].

Spin-exotic hybrid mesons have gluonic components with non-trivial angular momentum. This can be modelled on a lattice by joining the quark and antiquark by a colour flux path (product of links) which is not straight. Guidance in designing suitable paths comes from the heavy quark studies where a U-shaped path (actually a difference of the form $\sqcap-\sqcup$ ) between static quarks was found [3, 4] to yield the lightest hybrid excitation. We construct hybrid meson operators for light quarks by joining the light quarks by colour flux in that U-shaped orientation. This involves an extra quark propagator inversion, but we are able to study a range of different hybrid mesons using this extra inversion. Effectively the technique is to employ a 'white' source on the lattice and to separate the hybrid state of interest by choosing an appropriate operator for the sink [8].

Details of the lattice evaluation and operator construction are given in ref. 88 and we summarise them here. We used a $16^{3} \times 48$ lattice at $\beta=6.0$ with the Wilson gauge action for SU(3) colour fields. This corresponds to a lattice spacing of approximately $0.1 \mathrm{fm}$. The quark propagators were evaluated at hopping parameter $K=0.137$ using an SW-clover fermionic action with coefficient $c=1.4785$. For our U-shaped source we took a separation of 6 lattice units and we evaluated 350 local propagators from sources at $(0,0,0,0)$ and at $(0,0,6,0)$. For the sink at 


\begin{tabular}{lllll}
$\begin{array}{l}\text { meson } \\
\text { multiplet }\end{array}$ & $J^{P C}$ & $\begin{array}{l}\text { mass } \\
M a\end{array}$ & $M / M_{V}$ & $M_{s} / M(\phi)$ \\
\hline$\hat{\rho}$ & $1^{-+}, 3^{-+}$ & $0.95(7)$ & $1.76(13)$ & $1.95(13)$ \\
$\hat{\mathrm{a}}_{0}$ & $0^{+-}, 4^{+-}$ & $1.05(7)$ & $1.94(13)$ & $2.16(13)$ \\
$\hat{\mathrm{a}}_{2}$ & $2^{+-}, 4^{+-}$ & $1.26(13)$ & $2.33(24)$ & $2.62(24)$ \\
$\mathrm{a}_{2}$ & $2^{+-}, 3^{+-}$ & $1.12(10)$ & $2.07(18)$ & $2.32(18)$
\end{tabular}

Table 1: The masses of the hybrid mesons. The $J^{P C}$ values are the lowest two values allowed by the lattice cubic symmetry. The masses are given in lattice units and as a ratio to the vector meson mass $m_{V}$. The mass ratio appropriate to $s \bar{s}$ states is given in the last column.

time $t$, we used U-shapes of size 1, 3 and 6 lattice units.

Using the same operator at source and sink (namely with size 6) gives an effective mass which is an upper bound on the ground state mass. In order to estimate this ground state mass, we evaluate the correlation between our source operator and all three sink operators since this data set allows us to estimate the excited state contribution and so extract the ground state mass by making stable two state fits to these correlations. The effective mass values for $\hat{\rho}$ are shown in the Figure. Given that the signal to noise ratio deteriorates rapidly with increasing time separation $t$, it is vital to use the small $t$ data to extract the ground state mass. We used correlated fits to as wide a range of $t$ as possible consistent with acceptable goodness of fit. A correlated 2-state fit to $t$-range 2-10 was found to give the most reliable determination of the ground state mass. The statistical error was evaluated using bootstrap. A systematic error from the uncertainty in this fitting procedure was determined by considering a range of other fits (by varying the $t$-range, fixing the excited state mass excitation to 1.5 lattice units, using a 1-state fit only, using an uncorrelated fit, fitting only subsets of the data). In each case this systematic error was found to be not larger than the statistical error quoted.

Our lattice results for the $1^{-+}, 0^{+-}$and $2^{+-}$exotic hybrid mesons are given in the Table. Note that correlation studied in the last row in the Table can have contributions from a nonexotic $b_{3}$ meson as well as the exotic $2^{+-}$, so it is only included for completeness. Our results are consistent with the assumption that the $1^{-+}$state is the lightest. Taking account of statistical correlations, we find that the $0^{+-}$state is heavier than the $1^{-+}$state with a significance of 1 standard deviation.

As a cross check we also studied these states using a loop of colour flux which starts and finishes at the same site to model the gluonic excitation at the source. This closed gluon loop with non-trivial colour has the advantage of creating hybrid states which acn be studied without any additional quark propagators beyond those usually used in hadron spectroscopy. The disadvantage is that it creates hybrid states with the quark and antiquark at the same location and this may be an untypical component of the wavefunction. For the $J^{P C}=1^{-+}$ 


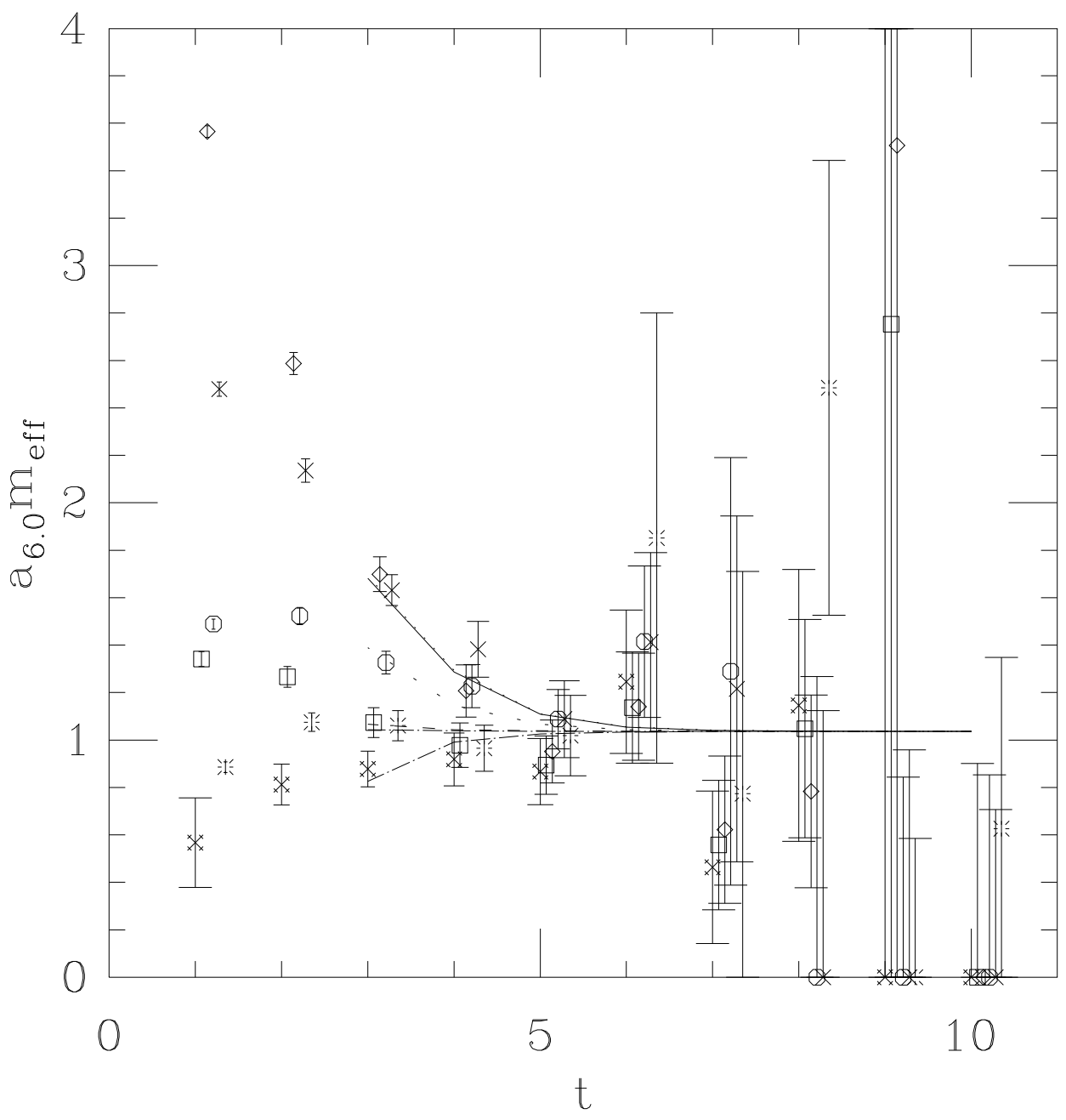

Figure 1: The lattice effective mass for the $J^{P C}=1^{-+}$hybrid meson versus time separation $t$. The source used was a U-shaped path of size $6 \times 6$, while the sinks were combinations of U-shaped paths of size $6 \times 6(\diamond), 3 \times 3(\square)$ and $1 \times 1$ (fancy cross). We also used closed colour loops at the source of size $6 \times 6(*), 3 \times 3$ (octagon) and $1 \times 1(\times)$ with the above sinks. The simultaneous two state fit to all six correlations is shown. 
state, we used a 'magnetic' colour loop $\left(L^{P C}=1^{+-}\right)$and, as shown in the Figure, the effective mass from measuring 294 configurations is consistent with that obtained from U-shaped sources. From a fit to the closed-loop source correlations only, we obtained $M a=1.12(8)$. For the other exotic states, the loop was taken to be in a $L^{P C}=1^{-+}$representation and the resulting correlations were very noisy compared to those obtained from U-shaped sources.

We express our lattice results for the hybrid masses as a ratio to the vector meson mass we find on the same lattices, namely $M_{V} a=0.54(1)$. Since for the hybrid mesons, we find $M a \approx 1$, we must address possible order $a$ errors which do not cancel in this mass ratio as $a$ is decreased to the continuum limit. The SW-clover fermionic action has been used with the intention of reducing such errors. Indeed a study [10] varying $a$, shows that dimensionless mass ratios are consistent with constant over a range of lattice spacings including that used here, unlike the case when the Wilson fermionic action is used. It is difficult to estimate the systematic error from this extrapolation to the continuum limit of our results without repeating the calculation at smaller $a$. It is plausible that it is no larger than the statistical errors we quote.

Another potential source of error is that we use a finite spatial volume of extent approximately $1.6 \mathrm{fm}$. Investigations using larger volumes have shown that this is large enough for conventional mesons. For glueballs, finite size effects are known to be unimportant for spatial extent greater than $1 \mathrm{fm}$. The static quark analysis of hybrid mesons, however, finds that the effective potential is rather flat versus inter-quark separation so that the wave function will be spatially extended [3, 4. This implies that hybrid mesons may be significantly more extended than conventional quark model mesons or glueballs. Without repeating the hybrid mass calculation for larger volumes, we are unable to quantify the magnitude of finite size effects.

For computational reasons, we have used a quark mass which is heavier than the strange quark mass in order to improve the signal to noise ratio. Ideally one should study hybrid mesons at a range of quark masses and interpolate to the experimentally relevant cases. Here we use instead a model assumption to make the small correction to our quark mass to bring it to the strange quark mass. This is not without some uncertainty as the model is based on experimental data whereas the lattice results apply to the quenched approximation - see ref. 11] for a discussion of this problem. In the quenched approximation, there will be magic mixing (i.e. no mixing) and the $s \bar{s}$ pseudoscalar will be at $0.68 \mathrm{GeV}$ : thus $M_{\phi} / M_{\eta(s)}=1.5$. We find $M_{V} / M_{P}=1.31(1)$ which implies that our quark mass is somewhat heavier than the strange quark. To correct for this, the simplest assumption, which is string inspired, is that for two types of quark $q_{1}$ and $q_{2}: M\left(q_{1}\right)^{2}-M\left(q_{2}\right)^{2}$ is the same for hybrid, vector and pseudoscalar mesons. This implies that if our lattice ratio is $M / M_{V}=x$ then for $s$-quarks $M_{s} / M_{\phi}=\sqrt{x^{2}+0.33\left(x^{2}-1\right)}$. This correction is shown in the Table.

Using the experimental $\phi$ meson mass to set the scale, our result for the lightest $s \bar{s}$ hybrid meson is 1.99(13) Gev. Taking account of some of the possible systematic errors described above, we round this prediction to $2.0(2) \mathrm{GeV}$. We can use the model described above to estimate that the corresponding non-strange hybrid meson would be $120 \mathrm{MeV}$ lighter than the $s \bar{s}$ state. 
It is interesting to compare our result with model estimates. There have been two classes of model which have been proposed to describe the hybrid spectrum. Constituent gluon models, of which the bag model [12] is the prototype, have a lowest gluonic excitation which is 'magnetic' with $L^{P C}=1^{+-}$. This implies that the $J^{P C}=1^{-+}$spin exotic hybrid will be lightest in constituent models. The spectrum of these 'constituent gluons' has been studied on a lattice [14] which confirms that the magnetic gluon is indeed the lightest such excitation. The other class of model treats the gluonic excitation as an excited state of a string or fluxtube, as motivated by the lattice description [3] of hybrid mesons with heavy quarks. A continuum version of this fluxtube model [13] has been used to give predictions for hybrid mesons containing light quarks. These levels are expected to be relatively heavy: about $2.1 \mathrm{GeV}$ for the $s \bar{s}$ system. This energy value comes basically from the string model which gives excited levels with energies $n \pi / R$ higher for integer $n$. Then $R$, which is the interquark separation, can be estimated from standard quark models. This fluxtube model leads to degenerate hybrid levels which include spin-exotic $J^{P C}=1^{-+}, 0^{+-}$, and $2^{+-}$. A reasonable compromise from these two models is that the fluxtube model would be a good approximation to the hybrid spectrum but that the degeneracy of the spin-exotic levels would be broken in the direction predicted by the constituent aproach. This is exactly what our results indicate.

A study of light-quark hybrid mesons needs input from the theoretical mass values in the quenched approximation to guide experimental searches and to provide input to models for the mixing between the $q \bar{q}$ hybrid states and $q \bar{q} q \bar{q}$ or two meson states. Here we provide the first results of such a study from first principles. We focus on the spin-exotic states to avoid mixing with $q \bar{q}$ mesons. For these, the $J^{P C}=1^{-+}$state is consistent with being the lightest. The mass value that we obtain is $2.0(2) \mathrm{GeV}$ for the $s \bar{s}$ state. It is plausible that this error estimate includes the systematic errors but these systematic errors can be fully controlled by extending the lattice study to larger lattices, smaller lattice spacings and several quark masses. Our result for the mass spectrum of spin-exotic hybrid mesons is comparable to that suggested by fluxtube models.

We acknowledge support from EPSRC grant GR/K/41663 to the UKQCD Collaboration. We thank Stephen Pickles and David Richards for their help in setting up the inversion codes for the shifted local propagators.

\section{References}

[1] UKQCD collaboration, G. Bali et al., Phys. Lett. B309 (1993) 378.

[2] H. Chen, J. Sexton, A. Vaccarino and D. Weingarten; Nucl. Phys. B (Proc. Suppl.) 34 (1994) 357;

J. Sexton, A. Vaccarino and D. Weingarten, Phys. Rev. Lett.75 (1995) 4563.

[3] L.A. Griffiths, C. Michael and P.E.L. Rakow, Phys. Lett. 129B (1983) 351; 
[4] S. J. Perantonis and C. Michael, Nucl. Phys. B347 (1990) 854.

[5] R. Sommer, hep-lat/9401037 (to be published in Phys. Rep.).

[6] R. M. Barnett et al., Phys. Rev. D54 (1996) 1.

[7] F. E. Close, Proc. Hadron Conference, Manchester 1995; hep-ph/9509245.

[8] UKQCD Collaboration, P. Lacock et al., Orbitally excited and hybrid mesons from the lattice, Phys. Rev. D (in press), hep-lat/9605025.

[9] C. Bernard et al., Exotic hybrid mesons with light quarks, hep-lat/9607031.

[10] UKQCD Collaboration, C. Michael and H. Shanahan, Nucl. Phys. B (Proc. Suppl.) 47 (1996) 337.

[11] UKQCD Collaboration, P. Lacock and C. Michael, Phys. Rev. D 52 (1995) 5213.

[12] R. L. Jaffe and K. Johnson, Phys. Lett. B 60 (1976) 201;

M. S. Chanowitz and S. R. Sharpe, Phys. Lett. B126 (1983) 225;

T. Barnes and F. E. Close, Phys. Lett. B116 (1983) 365.

[13] N. Isgur and J. Paton Phys. Lett. B124 (1983) 247; Phys. Rev. D 31 (1985) 2910.

[14] I.H. Jorysz and C. Michael, Nucl. Phys. B302 (1988) 448;

N.A. Campbell et al., Phys. Lett. 167B (1986) 91;

M. Foster and C. Michael (in preparation). 